\title{
A HIGHER ORDER NONLINEAR SCHRÖDINGER EQUATION
}

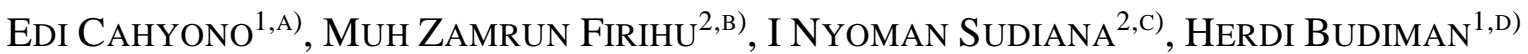 \\ AND MUH KABIL DJAFAR ${ }^{1, E)}$ \\ 1 Department of Mathematics FMIPA Universitas Halu Oleo \\ Jl. HEA Mokodompit, Kendari, 93232 Indonesia \\ 2 Department of Physics FMIPA Universitas Halu Oleo \\ Jl. HEA Mokodompit, Kendari, 93232 Indonesia
}

A)Corresponding author: edi_cahyono@innov-center.org, edi.cahyono@uho.ac.id

B) mzamrun@gmail.com

C) sudiana75@yahoo.com

D) herdi_budiman@yahoo.com

E) kabildjafar@gmail.com

\begin{abstract}
Nonlinear Schrödinger (NLS) equation has been widely studied, and it has been appeared in tremendous amount of papers. NLS equation models a wave packet travelling in dispersive and nonlinear media. In this paper, a higher order NLS equation is discussed. The solution, which is complex wave envelope, is investigated numerically for narrow and broad envelope. Broader envelope shows deformation during the evolution, while narrow envelope does not. Another finding is that the fifth order nonlinearity does not contribute significantly to the envelope deformation. Hence, working with higher order will take much effort but insignificant results.
\end{abstract}

Keywords: Dispersive Equation, Linear Envelope Equation, Nonlinear Schrödinger (NLS) equation

\section{Introduction}

Nonlinear Schrödinger (NLS) equation has been widely studied, and it has been appeared in tremendous amount of papers, from theory to applications. Among others are [1, 2, 3, 4]. For application in quantum mechanics, NLS type of equations were discussed in $[5,6]$, and in $[7$, 8] NLS type of equations were used to study Bose-Einstein condensate theory. For application in photonics, that is a study of light or photon generation and the manipulation of light and photon generation, NLS equation can be found in [9] or more recently [10]. NLS equation also appeared to study plasma, one of the four fundamental states of matter (solid, liquid, gas and plasma), [11, 12]. NLS equation also has been applied in the study of molecular dynamics, that is a study of the motion of atoms, molecules and for particle accelerator [13, 14], including for bio-molecule dynamics $[15,16]$. Applications in semiconductor electronics have been discussed by Brezzi and Markowich [17], and Lopez and Soler [18].

From mathematical point of view and theoretical wave, NLS equation is the evolution of a wave group where the waves are dispersive and nonlinear. A simple model of dispersive and nonlinear wave is in the form of Korteweg-de Vries (KdV) equation [19]. An elegant derivation by applying Hamiltonian system was presented in [20]. A KdV type by applying exact 
dispersion relation has been discussed in [21]. For more general dispersion and second order nonlinearity, a derivation has been presented in [22] which resulted in a KdV type of equation represented in a pseudo derivative for dispersive term and pseudo nonlinear for the second order nonlinear term. The relation between KdV equation and NLS equation has been discussed in $[22,23]$. It is related with the so-called side band interactions [22, 23, 24], especially the third order interaction and higher interactions.

This paper is intended to investigate the effect of the nonlinearity of NLS, both the third order and the fifth order nonlinearity independently as well as altogether. It is also to understand the relation of the effect with the solution properties of dispersive and nonlinear equation and/or the phenomena of dispersive and nonlinear wave. This paper is a continuation of [24].

\section{Dispersive Equation and Linear Envelope Equation}

A well-known dispersive and nonlinear equation is the KdV equation [19]. In the standard form, $\mathrm{KdV}$ equation may be found in many standard text books on water wave theory [25, 26]. This paper considers a more general dispersive and nonlinear equation in the form

$$
u_{t}+i \Omega\left(-i \partial_{x}\right) u+b u u_{x}=0
$$

where $\Omega(k)=k \sqrt{\frac{\tanh k}{k}}$ which is called exact dispersion relation. Equation (1) has appeared in several papers such as [24, 27, 28], after it was introduced in [21] and re-derived in [22]. Approximating up to third order of $k, \Omega(k) \approx k-\frac{1}{6} k^{3}$. Applying this approximate dispersion relation and $b=\frac{1}{3},(1)$ becomes

$$
u_{t}+u_{x}+\frac{1}{6} u_{x x x}+\frac{1}{3} u u_{x}=0
$$

Linear part of (1) is merely dispersion equation dispersive and nonlinear equation in the form

$$
u_{t}+i \Omega\left(-i \partial_{x}\right) u=0
$$

Let $\exp (i(k x-\Omega(k) t))$ satisfies (3), hence, the exact dispersion relation is satisfied for any $k$. The sum for any $k$ is also satisfies (3)

$$
u(x, t)=\int a(k) \exp (i(k x-\Omega(k) t)) d k+c . c .
$$

where c.c. stands for complex conjugate. Writing

$$
\begin{aligned}
A(x, t) & =\int a(k) \exp (i((k-\bar{k}) x-(\Omega(k)-\Omega(\bar{k})) t)) d k \\
& =\int a(\bar{k}+\kappa) \exp (i((\kappa) x-(\Omega(\bar{k}+\kappa)-\Omega(\bar{k})) t)) d \kappa,
\end{aligned}
$$

eqn (4) can be written in the form

$$
u(x, t)=A(x, t) \exp (i(\bar{k} x-\Omega(\bar{k}) t))+c . c .
$$

Often, $\exp (i(\bar{k} x-\Omega(\bar{k}) t))$ is called as complex carrying wave and $A(x, t)$ is the corresponding complex envelope. Figure 1 illustrates the (carrying) wave and its corresponding envelope. 


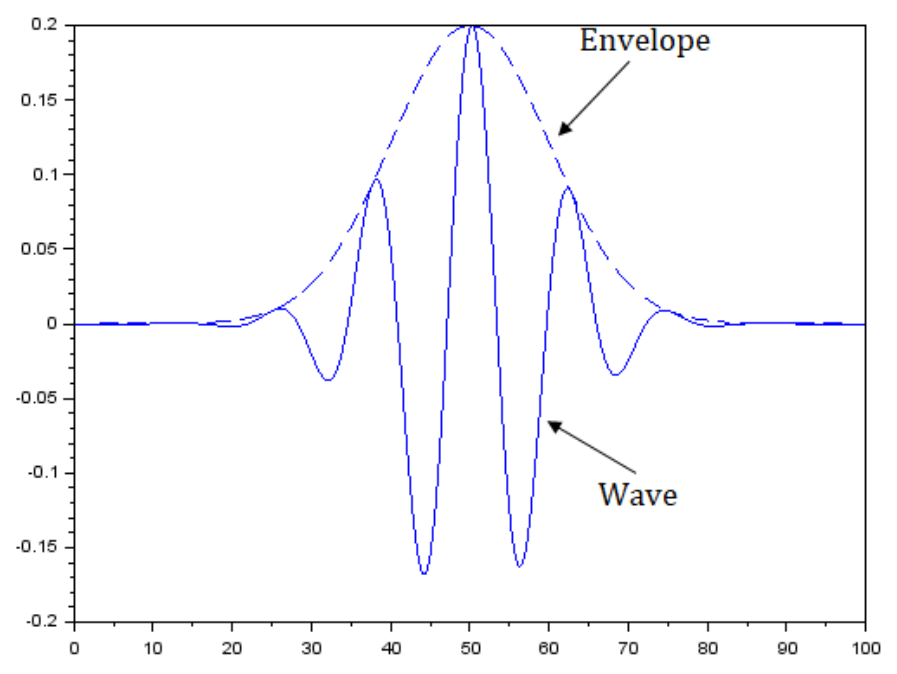

FIGURE 1. A (carrying) wave and its corresponding envelope.

Applying transformation $t=\tau$ and $\xi=x-\Omega^{\prime}(\bar{k}) t, A$ of eqn (5) satisfies

$$
A_{\tau}+i \Omega_{1}(-i \partial \xi) A=0
$$

where $\quad \Omega_{1}(\kappa)=\Omega(\bar{k}+\kappa)-\Omega(\bar{k})-\Omega^{\prime}(\bar{k}) \kappa \quad$. Often, $\quad \Omega_{1}(\kappa) \approx \frac{1}{2} \Omega^{\prime \prime}(\bar{k}) \kappa^{2}$. For this approximation, (6) becomes

$$
A_{\tau}+i \beta A_{\xi \xi}=0
$$

where $\beta=-\frac{1}{2} \Omega^{\prime \prime}(\bar{k})$. Eqn (8) is the linear part of the well-known NLS equation.

\section{Qubic Quintic NLS Equation}

A standard Nonlinear Schrödinger (NLS) equation is in third order nonlinearity. A higher order NLS equation usually in third order and fifth order nonlinearity. For derivation of NLS equation, one may read [1], or [22, 23] for a more general dispersion relation. Consider a higher order NLS equation in the form

$$
A_{\tau}+i \beta A_{\xi \xi}+i \gamma_{1} A|A|^{2}+i \gamma_{2} A|A|^{4}=0
$$

where $\beta, \gamma_{1}$ and $\gamma_{2}$ are constant.

In this section, numerical solutions are sought. Direct Euler method has been applied for the derivative of time, and central difference has been applied for second order of space. For the need of numerical computation, pseudo boundary condition is considered, where the boundary is approximated with a quadratic function by applying three nearest points (inside the domain) to the boundary as illustrated in Figure 2. Spatial grid has been set to be 0.1, and the time set has been 0.01 . The initial condition is

$$
f(\xi)=a \exp \left(-b(\xi-c)^{2}\right)
$$

where $a, b$ and $c$ are constant. For all computation, $a=0.75$ and $c=50$. 


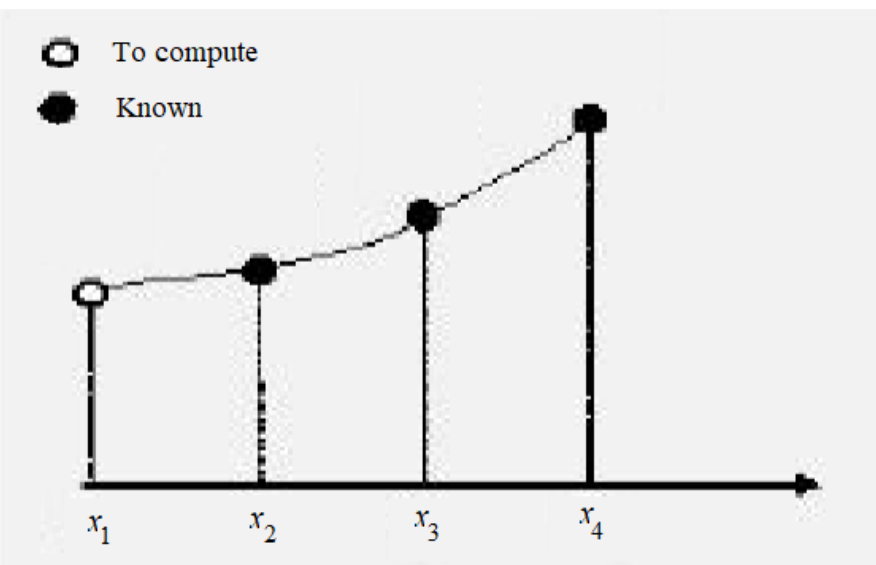

FIGURE 2. A quadratic approximation of the boundary by applying three nearest points.

Figure 3 shows the evolution of complex amplitude $A$ for various value of $b$. Parameter $b$ related to the narrowness of the complex envelope $A$. The larger $b$, the narrower $A$. On the other hand, the narrowness of complex envelope is related to the narrowness of complex wave spectrum, see $[22,23]$. On the opposite, the narrower complex envelope, the broader the complex wave spectrum. Figure 3 shows that the broader complex envelope develops deformation during the evolution, figure (a). On the other hand, the narrow complex envelopes do not develop deformation, figure (b), (c) and (d). This finding has a good agreement with previous experiment, and simulation of surface water waves such as reported in [29. 30, 31, 32].

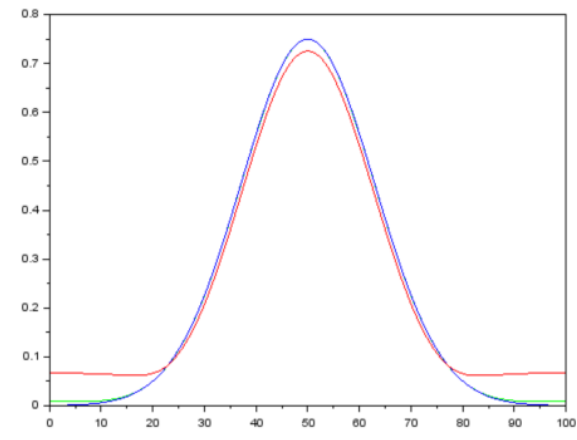

(a) $b=0.003$

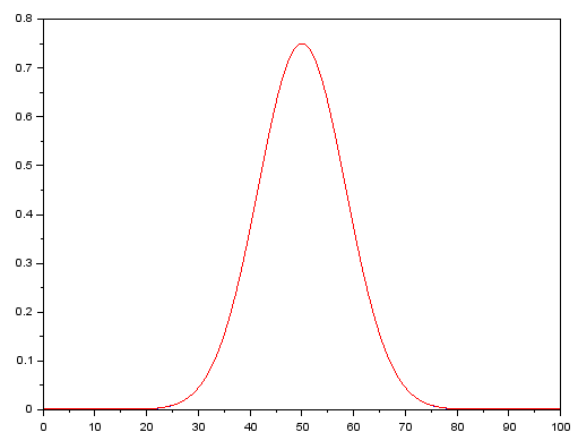

(c) $b=0.007$

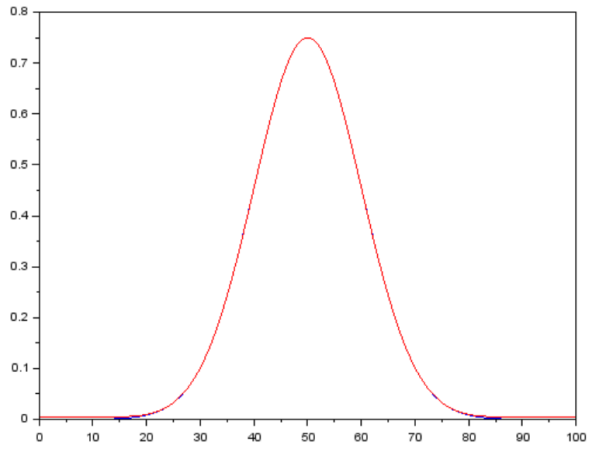

(b) $b=0.005$

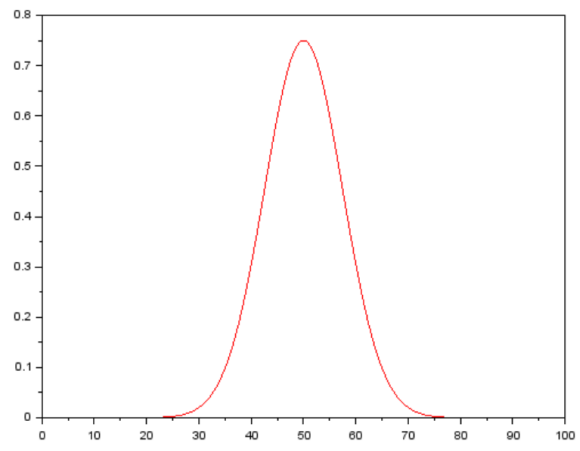

(d) $b=0.009$

FIGURE 3. Evolution of complex envelope $A$ at $\tau=0 \mathrm{t} 1=0$ (blue), $\tau=1.75$ (green), $\tau=2.25$ (red). 
Figure 4 shows the effect of nonlinearity to the evolution of broad complex envelope $A$. The plots are for $\tau=0$ and $\tau=2.25$ to show the nonlinear contribution after interval of time of evolution. Without the nonlinearity, it is hardly to see the envelope deformation. While the effect of nonlinearity is very recognizable, although it is still difficult to observe the significant the effect of the fifth order nonlinearity (compare red from green).

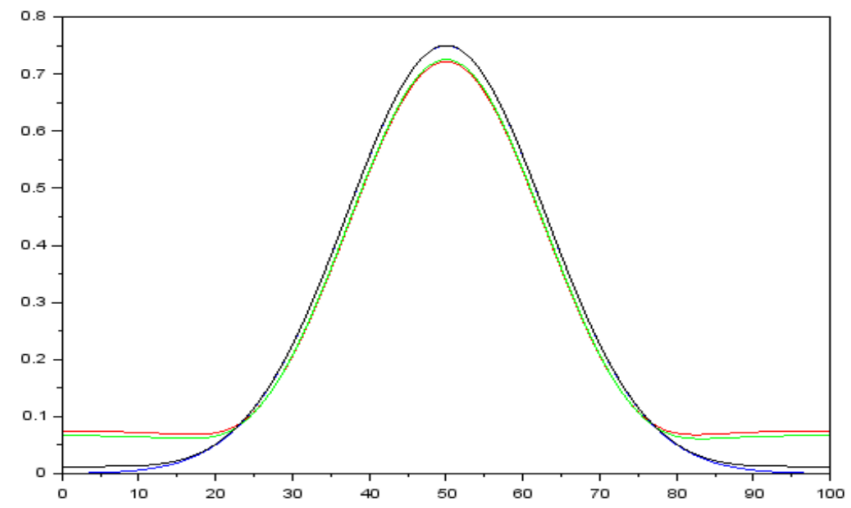

FIGURE 4. Contribution on nonlinearity. $\tau=1.75$ and $\tau=2.25$. Initial condition (blue), linear (black), 2nd order (green), 3rd order (red).

\section{Conslusion and Future Research}

This paper discussed complex carrying wave which was a solution of dispersive and nonlinear equation, and the corresponding complex envelope which satisfies NLS equation. The solutions of NLS equation were obtained numerically, and they were graphically presented for narrow and broad envelopes. It was observed that broad complex envelope developed deformation during the evolution, but the narrow one did not. And, this agrees with the previous finding for corresponding complex carrying wave, numerically as well as experimentally.

Nonlinearity contribution to the solution, or envelope deformation was clearly observed for broader complex envelope. However, it was still difficult to separate the fifth order contribution together from the third order one. It indicates that the fifth order contribution is very small. Hence, working with third order contribution is more desirable than with higher order. It is because the simplicity, and there is no much different of the results from the higher order equation.

It is noted that the third order nonlinearity of NLS equation obtained from dispersive and second order nonlinearity equation. There is possibility that the third order nonlinearity of the dispersive and nonlinear equation significantly contributes to the third order nonlinearity of NLS equation. Future research will be focused on dispersive and third order nonlinear wave equation and the relation to NLS equation.

\section{Acknowledgments}

This research has been supported by DRPM Kemenristekdikti, Indonesia via hibah Penelitian Dasar Unggulan Perguruan Tinggi (PDUPT) 2017 - 2019 [Contract No. 621/UN29.20/PPM/2017, Contract No. 448/UN29.20/PPM/2018 and Contract No. 515e/UN29.20/PPM/2019]. 


\section{Refferences}

[1] J. P. Boyd and G-Y. Chen, Math. Comput. Simulat. 55, 317-328 (2001).

[2] V. Vyas, T. S. Raju, N. K.Choragudi and P. K. Panigrahi, J. Phys. A: Math. Gen. 39, 9151 (2006).

[3] G. Amador, K. Colon, N. Luna, G. Mercado, E. Pereira and E. Suazo, Simetry 8(38), 116 (2016).

[4] A. Houwe, M. Justin, D. Jerome, G. Betchewe, S. Doka and K. T. Crepin, J. Phys. Commun. 3011002 (2019).

[5] J. L. Rosales, and J. L. Sanchez-Gomez, Phys. Lett. A, 166, pp 111-115 (1992).

[6] Q. Zhang and T. Ma, J. Appl. Math. Article ID 387823, 15 pages, http://dx.doi.org/10.1155/2012/387823 (2012).

[7] F. Dalfovo, S. Giorgini, L. P. Pitaevskii, and S. Stringari, Rev. Mod. Phys. 71, pp. 463512 (1999)

[8] Y. Yıldırım, N. Çelik and E. Yaşar, Results in Physics, Nonlinear Schrödinger equations with spatio-temporal dispersion in Kerr, parabolic, power and dual power law media: A novel extended Kudryashov's algorithm and soliton solutions, 7, Pages 3116-3123 (2017).

[9] A. Hasegawa, Optical solitons in fiber (Springer-Verlag, Berlin, 1989).

[10] A. V. Dorofeenko, A. A. Zyablovsky, A. A. Pukhov, A. A. Lisyansky and A. P. Vinogradov, Phys. Uspekhi 55 (11) 1080 - 1097 (2012).

[11] R.K. Dodd, j. C. Eilbeck, J. D. Gibbon and H. C. Morris, Solitons and Nonlinear wave Equations, (Academic Press, New York, 1982).

[12] K. Baumgärtel and K. Sauer, Topic on Nolinear Wave-Plasma Interaction, (Birkhäuser Verlag, Stuttgart, Germany, 1987).

[13] R. Fedele, G. Miele, L. Palumbo and V. G. Vaccaro, Phys. Lett. A, 173, pp 407-413 (1993).

[14] A. Kundu and T. Naskar, Phys. D 276, 21-27 (2014).

[15] A. S. Davydov, Solitons in moleculer systems, (Reidel, Dordrecht, 1985).

[16] M. Peyrard, Nonlinear Exitation in Biomolecules, (Springer-Verlag, Berlin, 1994)

[17] F. Brezzi and P. A. Markowich, Math. Model Methods Appl. Sci., 14 (1991), pp. 35-61 (1991).

[18] J. L. Lopez and J. Soler, Math. Model Methods Appl. Sci., 10, pp. 923-943 (2000).

[19] D. J. Korteweg and G. de Vries, Philosophical Magazine, 39(240):422-443, (1895) doi: 10.1080/ 14786449508620739

[20] E. van Groesen and S. R. Pudjaprasetya, Wave Motion 18: 345-370 (1993).

[21] E. van Groesen, J. Eng. Math. 34: 215-226 (1998).

[22] E. Cahyono, Analytical Wave Codes for predicting surface waves in a laboratory basin (PhD thesis University of Twente, Enschede, 2002).

[23] E. van Groesen, E. Cahyono and A. Suryanto, Optic. Quantum Elect. 34, 577-595 (2002).

[24] E. Cahyono, L. D. Ngkoimani and M. Ramli, Intl J. Math. Anal. 9(43), 2121 - 2132 (2015).

[25] E. Cahyono, I N. Sudiana, R. Raya and M. Z. Firihu, J. Phys.: Conf. Ser. 1218012002 (2019)

[26] G. B. Whitham, Linear and nonlinear waves (John Wiley \& Sons, New York, 1974) pp. 460-470.

[27] L. Debnath, Nonlinear water waves (Academic Press Inc, San Diego, 1994).

[28] M. Ramli, S. Munzir, T. Khairuman and V. Halfiani, Far East J. Math. Sci, 93 no. 1, 97 - 117 (2014).

[29] Afriadi, Y., Zahara, V., Halfiani, H. A., Mardi and M., Ramli, J. Phys.: Conf. Ser.1116 022002 (2018). 
[30] C. T. Stansberg "On the nonlinear behaviour of ocean wave groups" in Ocean Wave Measurement and Analysis Edited by B. L. Edge and J. M. Hemsley, (American Society of Civil Engineers (ASCE) 2, Reston, VA, 1997), pp. 1227-1241.

[31] J. Westhuis, E. van Groesen and R. H. M. Huijsmans, J. Waterway, Port, Coast Ocean Eng. 127(6):334-342 (2001).

[32] J. Westhuis, The numerical simulation of nonlinear waves in a hydrodynamic model test basin (PhD thesis University of Twente, 2001). 